\title{
Expression Patterns of Snail1, E-Cadherin and N-Cadherin Proteins in the Developing Kidney and in Wilms' Tumor (Nephroblastoma)
}

\author{
Sebastian Christoph Schmid • Kareen Blechschmidt • \\ Stefan Schulz $\cdot$ Karl Friedrich Becker $\cdot$ Gregor Weirich
}

To view enhanced content go to www.rarecancers-open.com

Received: May 1, 2014 / Published online: July 16, 2014

(C) The Author(s) 2014. This article is published with open access at Springerlink.com

\section{ABSTRACT}

Introduction: Snail1 is a transcription factor that suppresses E-cadherin. The roles of Snail1 and E-cadherin in mesenchyme-epithelial transition (MET) are not yet clear. We explored the role of Snail1, E-cadherin and N-cadherin in fetal kidneys and compared it with Wilms' tumor/ nephroblastoma (WT), a rare mesenchymal kidney tumor, to better understand MET in organogenesis and malignancy.

Methods: A total of 19 samples were analyzed, consisting of seven childhood WT, two adult WT and ten fetal kidneys. Immunohistochemistry was used to analyze Snail1, E-cadherin, and $\mathrm{N}$-cadherin protein expression in the samples as well as their cellular localization.

Electronic supplementary material The online version of this article (doi:10.1007/s40487-014-0005-0) contains supplementary material, which is available to authorized users.

S. C. Schmid ( $\square)$

Department of Urology, Technische Universität

München, Munich, Germany

e-mail: sebastian.schmid@1rz.tum.de

K. Blechschmidt · S. Schulz · K. F. Becker .

G. Weirich

Institute of Pathology, Technische Universität

München, Munich, Germany
Results: Six of the childhood WT showed a cytoplasmic Snail1 reaction. Adult WT were Snail1 negative. E-cadherin expression was found in epithelial structures in eight of the nine WT. Tubular epithelial cells showed a Snail1 positivity in the cytoplasm of seven kidneys. E-cadherin expression was found in epithelia of all ten fetal kidneys, but not in the mesenchyme. Four out of ten fetal kidneys and six out of nine WT were N-cadherin positive. One out of ten fetal kidneys showed a nuclear expression of Snail1 in undifferentiated mesenchymal cells.

Conclusions: Snail1 seems to play a role in kidney differentiation and in childhood WT. Our findings do not support the assumption that WT recapitulate renal organogenesis, at least not with respect to distribution patterns of cadherins and Snaill.

Keywords: E-cadherin; Kidney; MET; Ncadherin; Nephroblastoma; Oncology; Snail; Snail1; Wilms' tumor

\section{INTRODUCTION}

Renal embryogenesis is characterized by the sequential development of an undifferentiated 
metanephric mesenchyme (MM) into a complex organ composed of specialized epithelial structures and stroma. This process is initiated by the ureteric bud (UB), an early epithelial structure derived from the Wolffian duct [1]. A crucial factor for the developing kidney is the product of Wilms' tumor suppressor gene 1 (WT1) especially with respect to mesenchymal-epithelial balancing $[2,3]$. WT1 seems to interact with Snail2 (slug), a close relative of Snail1 [4]. With ongoing development the UB invades the MM and prevents the MM from entering apoptosis. The MM differentiates along several lines giving rise to stem cells, stroma and epithelial cells. The morphologic development of epithelia begins with the formation of condensation areas, evolving into intermediate structures (comma-shaped bodies, then s-shaped bodies) that in turn give rise to nephrons. Major proteins that participate in the generation and maintenance of epithelia in the developing kidney are members of the cadherin superfamily. Cadherins are cell adhesion molecules typically forming homotypic and homophilic junction type complexes. Cadherins are involved in intracellular signaling and the type I cadherins, E-cadherin and $\mathrm{N}$-cadherin, seem to play major roles in renal epitheliogenesis [5]. E-cadherin is a transmembrane protein encoded by the $\mathrm{CDH} 1$ gene at chromosome 16q22 and expressed in epithelial cells. The intracellular interaction of this protein with beta-catenin is essential for cell adhesion [6]. The expression of E-cadherin is influenced by several factors, amongst others the transcriptional regulator Snail1, and Snail2 (slug). Snail1, a zinc finger protein is a member of the snail superfamily [7]. Studies with snail1-/-mice indicate that this protein is a central regulator of gastrulation and is crucial for development as snail1-/- mice die early during embryonic development [8]. Snail1 was shown to induce transcription of genes linked to mesodermal differentiation like vimentin and fibronectin. Snail1 also acts as a transcriptional suppressor of E-cadherin, cytokeratin 18, claudin and occludin. In turn, Snail1 is regulated by various growth factors, namely fibroblast growth factor, endothelial growth factor, tumor growth factor- $\beta$, bone morphogenetic protein and Wingless (WNT), which are all crucial for embryonic development [9]. Snail2 (slug) is considered a functional equivalent of Snail1 but can be induced by the latter [10]. Active Snail1 is localized in the nucleus, whereas inactive Snail1 transfers to the cytoplasm for ubiquitination and degradation [11, 12]. Snail is a putative inducer of mesenchymal-epithelial transition (MET)/epithelial-mesenchymal transition (EMT), cell movement and survival [13]. During embryonic development, epithelial and mesenchymal phenotypic transitions, as described above, are necessary for the formation of different tissues. However, these processes are not only involved in development but they seem also to be implicated in pathologic processes such as malignancy and fibrosis [10]. A characteristic point of EMT is the mutual interplay of Snail1 and E-cadherin, where Snail1 induces a reversal from the epithelial phenotype (characterized by E-cadherin expression) to a mesenchymal phenotype (characterized by $\mathrm{N}$-cadherin and vimentin expression among others) [14]. On the other hand, much less is known about the regulators that govern MET, which is probably due to the fact that morphologic evidence of MET has mainly been produced for the organogenesis of the kidney and testis [1]. However, in Wilms' tumor (WT), a human kidney neoplasia mainly occurring in infants, it has been speculated that an incomplete MET represents the initial 
step of neoplastic growth, recapitulating in part the morphologic pathway of kidney embryogenesis.

The aim of this study was to assess the expression of Snail1, E-cadherin and N-cadherin in the developing kidney to understand their participation in MET and to compare it with WT, a rare malignant renal tumor that exhibits morphologic similarities with the developing kidney.

\section{METHODS}

\section{Tissue Samples}

Nineteen formalin-fixed, paraffin-embedded surgical specimens were retrieved from the archives of the Institute of Pathology, Technical University Munich, Germany, and from the Institute of Pathology, University of Gießen, Germany. All procedures followed were in accordance with the ethical standards of the responsible committee on human experimentation (institutional and national) and with the Helsinki Declaration of 1975, as revised in 2000 and 2008. Informed consent was obtained from all patients for being included in the study. The samples consisted of ten fetal kidneys (12th week to 33rd week of gestation), seven childhood WT, and two adult WT. We used the Sn9H2 antibody, which was developed in house and tested for specificity [15]. Human placenta served as a positive control for all immunoreactions.

Three micrometer sections were taken from each block and transferred to coated slides. For anti-snail1 immunohistochemistry (IHC), sections were deparaffinized in graded alcohol and xylene and washed with phosphate buffered saline (PBS, pH 7.2-7.4). For antigen retrieval, sections were placed in a pressure cooker containing $0.01 \mathrm{Mol}$ citrate buffer with $0.1 \%$ Tween ( $\mathrm{pH}$ 6.0) for $4 \mathrm{~min}$.

Subsequently, sections were blocked with $200 \mu \mathrm{l}$ goat serum for $20 \mathrm{~min}$ and with an avidin-biotin complex (Avidin/Biotin Blocking Kit, Vector Laboratories, Burlingame, USA), followed by an incubation with rat primary antibody (Sn9H2) diluted 1:3,000 in PBS with $1 \%$ bovine serum albumin (BSA) for $2 \mathrm{~h}$ and then rinsed in PBS. Sections were then incubated with anti-rat secondary antibody (Vectastain Elite ABC Kit, Vector Laboratories, Burlingame, USA) diluted 1:200 in 1\% BSA PBS for $30 \mathrm{~min}$ and rinsed in PBS. After a 30-min incubation with the avidin-biotin peroxidase complex (Avidin/Biotin Blocking Kit, Vector Laboratories, Burlingame, USA) diluted 1:50 in PBS, diaminobenzidine and urea-hydrogen peroxide (Sigma-Aldrich, St. Louis, USA) were added for $10 \mathrm{~min}$ and rinsed in PBS. Finally, sections were counterstained with hematoxylin for $10 \mathrm{~s}$, and dehydrated in graded alcohol and xylene.

For anti-E-cadherin and anti-N-cadherin IHC, endogenous peroxidase was blocked with $1 \% \mathrm{H}_{2} \mathrm{O}_{2}$ for $15 \mathrm{~min}$ and then rinsed in PBS, followed by an avidin-biotin block (Avidin/ Biotin Blocking Kit, Vector Laboratories, Burlingame, USA). Sections were then incubated for $1 \mathrm{~h}$ with the primary murine N-cadherin antibody (clone 32, BD Transduction Laboratories, Franklin Lakes, USA) diluted 1:2,000 in PBS with 1\% BSA.

For anti-E-cadherin immunostaining, sections were incubated with the primary murine antibody against E-cadherin (clone 36, Transduction Laboratories, Franklin Lakes, USA) for $1 \mathrm{~h}$, diluted 1:1,500 in PBS with 1\% BSA. For both E- and N-cadherin immunostaining, sections were then incubated with an antimouse secondary antibody (Vectastain Elite ABC Kit, Vector Laboratories, Burlingame, 
USA), diluted 1:200 in 1\% BSA PBS for $30 \mathrm{~min}$, then rinsed in PBS. Following an incubation with the avidin-biotin peroxidase complex (Avidin/Biotin Blocking Kit, Vector Laboratories, Burlingame, USA) 1:50 in PBS for $30 \mathrm{~min}$, diaminobenzidine and urea-hydrogen peroxide (Sigma) were added for $10 \mathrm{~min}$ and sections were then rinsed in PBS. Eventually, sections were counterstained with hematoxylin for $10 \mathrm{~s}$ and dehydrated in graded alcohol and xylene.

\section{RESULTS}

\section{Snail1}

One out of ten cases showed a strong nuclear reactivity in stromal and some blastematous cells (Fig. 1a, b; Table 1). Seven out of ten fetal kidneys exhibited a cytoplasmic reactivity in tubular epithelial structures (Fig. 2). Mesodermal and stromal cells were not immunoreactive.
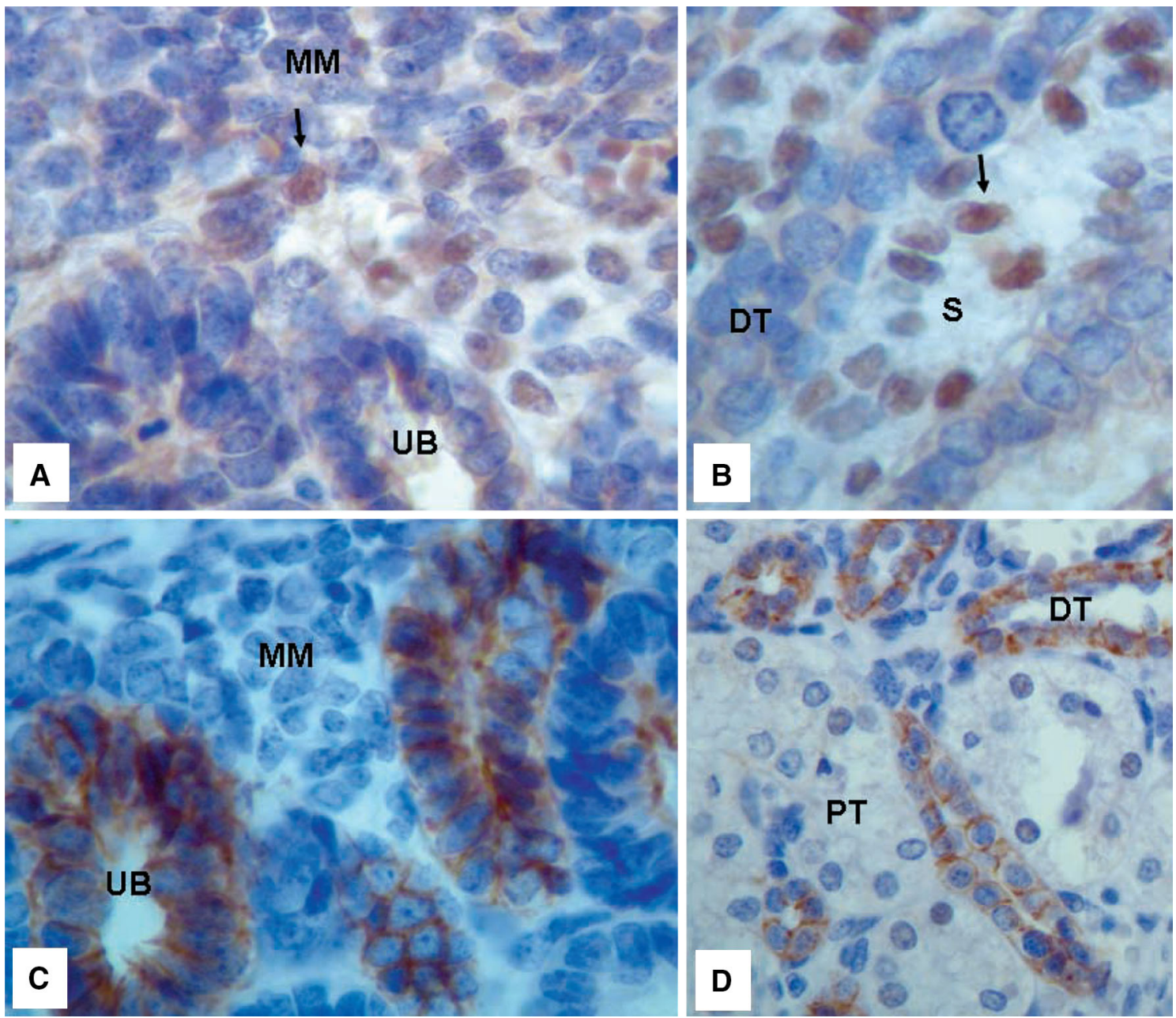

Fig. 1 Fetal kidney case number 7. a ( $\times 400)$ : Snail1positive nuclei of MM (arrow). Nuclei of UB are Snail1negative; b $(\times 400)$ : Stromal cells exhibit Snail1-positive nuclei, DT are Snaill-negative; c $(\times 400)$ : E-cadherin- positive cells of the UB, MM cells are negative; $\mathbf{d}(\times 200)$ : DT are E-cadherin-positive, PT are mainly E-cadherinnegative. $D T$ distal tubule, $M M$ metanephric mesenchyme, $P T$ proximal tubule, $S$ stroma, $U B$ ureteric bud 
Table 1 Results of immunohistochemical analysis

\begin{tabular}{|c|c|c|c|c|}
\hline Sample number & Age & Snail1 & E-cadherin & $\mathrm{N}$-cadherin \\
\hline \multicolumn{5}{|l|}{ Fetal kidneys } \\
\hline 1 & GW 22 & Negative & Positive & Negative \\
\hline 2 & GW 17 & Negative & Positive & Negative \\
\hline 3 & GW 33 & Positive (cytoplasmic) & Positive & Negative \\
\hline 4 & GW 26 & Positive (cytoplasmic) & Positive & Negative \\
\hline 5 & GW 12 & Positive (cytoplasmic) & Positive & Negative \\
\hline 6 & GW 14 & Positive (cytoplasmic) & Positive & Negative \\
\hline 7 & GW 19 & Positive (nuclear) & Positive & Positive \\
\hline 8 & GW 21 & Positive (cytoplasmic) & Positive & Positive \\
\hline 9 & GW 21 & Positive (cytoplasmic) & Positive & Positive \\
\hline 10 & GW 26 & Positive (cytoplasmic) & Positive & Positive \\
\hline \multicolumn{5}{|l|}{ Wilms' tumors } \\
\hline 1 & Childhood & Positive (cytoplasmic) & Negative & Negative \\
\hline 2 & Childhood & Positive (cytoplasmic) & Positive & Positive \\
\hline 3 & Childhood & Positive (cytoplasmic) & Positive & Positive \\
\hline 4 & Childhood & Negative & Positive & Negative \\
\hline 5 & Childhood & Positive (cytoplasmic) & Positive & Positive \\
\hline 6 & Childhood & Positive (cytoplasmic) & Positive & Positive \\
\hline 7 & Childhood & Positive (cytoplasmic) & Positive & Positive \\
\hline 8 & Adult & Negative & Positive & Positive \\
\hline 9 & Adult & Negative & Positive & Negative \\
\hline
\end{tabular}

$G W$ gestational week

In childhood WT, six out of seven tumors were positive. Three of the positive tumors exhibited a cytoplasmic Snail1 reaction in tubular structures, whereas nuclei remained negative. Stromal and blastematous cells were negative in these cases.

In the remaining three positive cases, blastematous areas were positive, but not stromal and tubular structures. Both adult WT were negative for Snail1 in stromal, tubular and blastematous areas.

\section{E-Cadherin}

Fetal kidneys showed an immunoreaction in distal parts of the nephron and the collecting duct, and a weak or no staining in the proximal tubules, glomeruli and blastematous cells (Fig. 1c, d; Table 1). Eight out of nine WT showed immunostaining of epithelial structures, whereas non-epithelial structures remained negative (Fig. 3c). Staining was strictly membrane bound. 


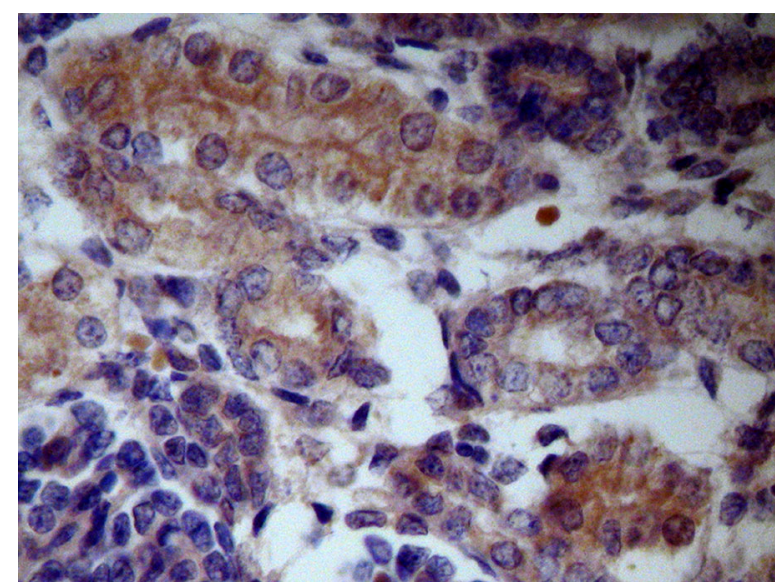

Fig. 2 Fetal kidney case number 9. $(\times 630)$ Snaill: proximal and distal tubules show cytoplasmatic staining. The glomerulum is not stained

\section{N-Cadherin}

Three out of ten fetal kidneys exhibited a weak immunoreaction, and one out of ten a strong cytoplasmatic immunoreaction in proximal tubular epithelial cells (Fig. 4). Blastematous and stromal cells were $\mathrm{N}$-cadherin negative. Six out of nine WT showed a similar reactivity in epithelial cells, whereas blastematous and stromal cells were negative (Fig. 3d).

Immunostains for cytokeratins and vimentin were used as marker reactions to verify an epithelial and/or mesenchymal differentiation, and were both positive in epithelial or mesenchymal structures in all fetal kidneys and all WT (data not shown).

\section{DISCUSSION}

We analyzed the expression patterns of Snail1, E-cadherin and $\mathrm{N}$-cadherin in fetal kidneys and WT in the context of MET to elucidate a putative biologic relationship between physiologic kidney development and neoplasia. The major limitation of the study is the small case number (aggravated by the rarity of WT) which makes statistical analysis pointless. Based on our finding that Snail1 was detectable in eight of the ten fetal kidneys, we speculate that Snail1 may be involved in MET of the developing kidney. However, only one out of ten kidneys exhibited a nuclear Snail1 immunostain (Fig. 1), whereas seven out of ten fetal kidneys showed a cytoplasmic anti-Snail1 reaction. This phenomenon may reflect the transition of active nuclear Snail1 to inactive cytoplasmic Snail1 prior to ubiquitination [5, 16-18]. Our finding of a predominant cytoplasmic Snail1 expression in most fetal kidneys between gestational weeks 12 and 33 may point to an early impact of Snail1 on kidney organogenesis, which is normally close to completion around gestational week 13. Our findings have recently been substantiated by Boutet et al. [10], who mapped Snail1 expression of murine kidneys to the pronephros state. Interestingly, in their study, Snail1 expression was followed by Snail2 (Slug) expression in the condensed MM surrounding the UB and Snail2 expression preceded cadherin expression (cadherin 16). We found this mutually exclusive expression pattern of Snail and E-cadherin only in adult WT in our study. Hence, our findings show the involvement of Snail1 and E-cadherin in renal organogenesis in humans. Further support for a putative role of Snail1 in kidney development stems from the experiments by Franci et al. [17], who found that Snail1 is an early and short-lasting marker of MET. The predominant expression of $\mathrm{N}$-cadherin in epithelia of the proximal nephron, where E-cadherin expression was not detected, points to a regulatory interplay of both proteins. Interestingly, an N-cadherin but not E-cadherin expression was also detected in proximal tubular epithelia of adult kidneys by Procialeck et al. [19]. This compartmentalized 

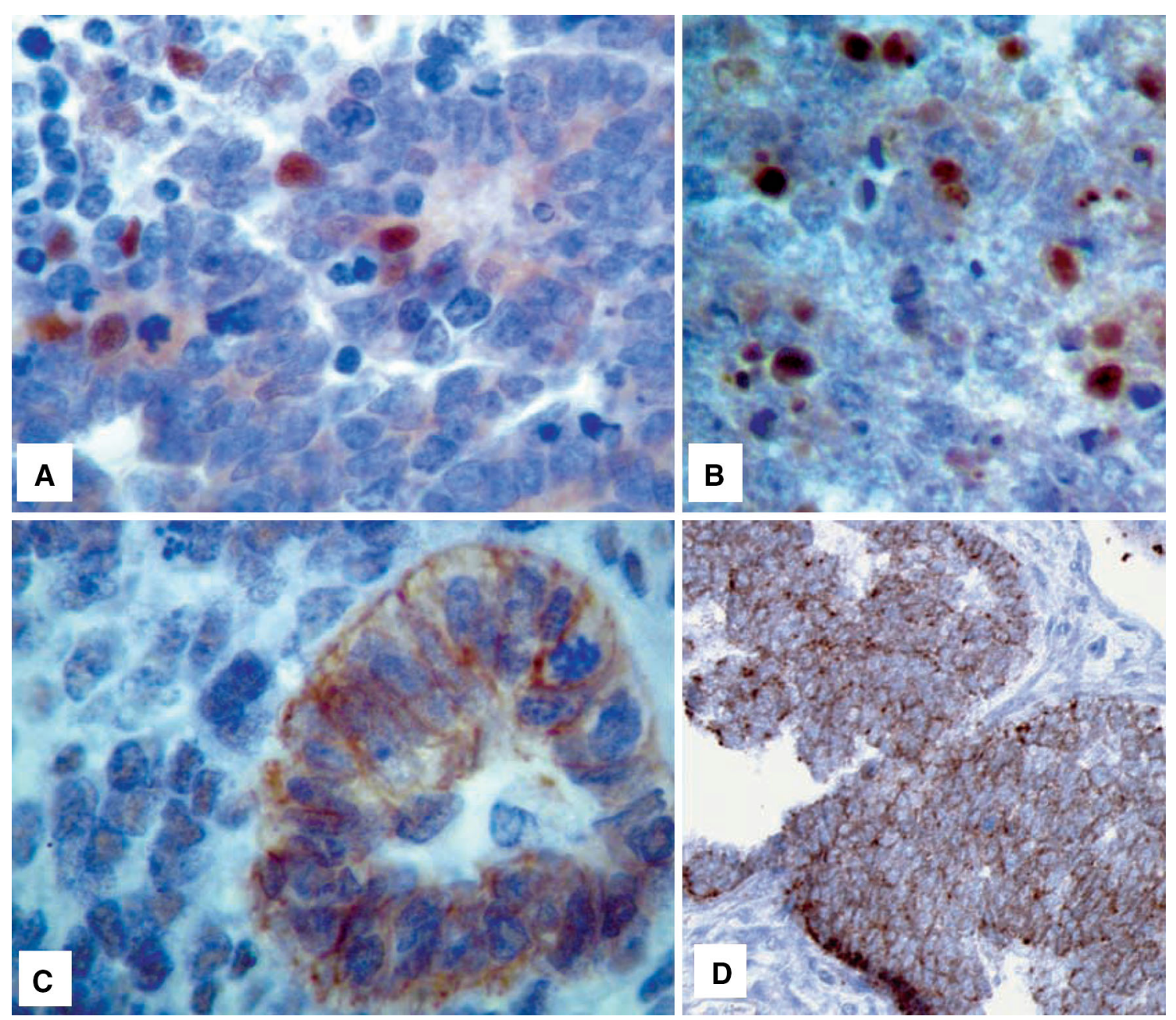

Fig. 3 Wilms' tumors. a $(\times 400)$ : Snail1: reactive nuclei can be seen in some primitive tubules; $\mathbf{b}(\times 400)$ : Snaill: reactive nuclei are present in blastematous cells; c $(\times 400)$ : E-cadherin: some tubules are positive for E-cadherin,

and long-lasting $\mathrm{N}$-cadherin expression (from gestational week 13 in our study to adulthood in Procialeck's study) may reflect an early need for a specific metabolic state that is maintained throughout life within this nephron segment.

The roles played by E-cadherin, N-cadherin and Snail1 in WT, however, seem less well organized as we found co-expression of both cadherins and Snail1 in some childhood WT. Obviously, these proteins may be activated in WT, but seemingly haphazardly. blastematous cells are Negative; d $(\times 200)$ : N-cadherin: some areas of undifferentiated blastema are strongly immunoreactive

\section{CONCLUSIONS}

Snail1, E-cadherin and N-cadherin are expressed in morphologically distinct patterns in the fetal kidney, although the main impact of Snail1 might occur earlier during embryogenesis. All three markers are also expressed in childhood WT, but apparently in a less organized pattern. Our findings do not support the assumption that WT recapitulate renal organogenesis, at least not with respect to the distribution patterns of cadherins and Snail1. 


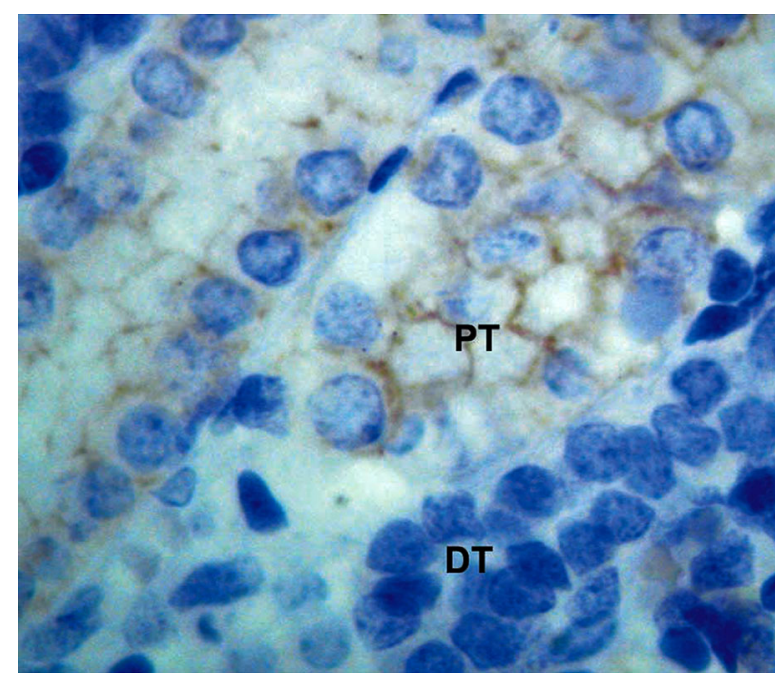

Fig. 4 Fetal kidney case number $7 .(\times 630)$ N-cadherin: proximal tubule cells are positive, distal tubule cells are negative

\section{ACKNOWLEDGMENTS}

We thank Prof. Dr. A. Schulz and Prof. Dr. Bohle for kindly providing archival material of Wilms' tumors. All named authors meet the ICMJE criteria for authorship for this manuscript, take responsibility for the integrity of the work as a whole, and have given final approval to the version to be published. No funding or sponsorship was received for this study or publication of this article.

\section{Conflict of interest. Sebastian Christoph} Schmid, Kareen Blechschmidt, Stefan Schulz, Karl Friedrich Becker and Gregor Weirich declare no conflict of interest.

Compliance with ethics guidelines. All procedures followed were in accordance with the ethical standards of the responsible committee on human experimentation (institutional and national) and with the Helsinki Declaration of 1975, as revised in 2000 and 2008. Informed consent was obtained from all patients for being included in the study.

Open Access. This article is distributed under the terms of the Creative Commons Attribution Noncommercial License which permits any noncommercial use, distribution, and reproduction in any medium, provided the original author(s) and the source are credited.

\section{REFERENCES}

1. Saxén L, Sariola H. Early organogenesis of the kidney. Pediatr Nephrol. 1987;1:385-92.

2. Donovan MJ, Natoli TA, Sainio K, et al. Initial differentiation of the metanephric mesenchyme is independent of WT1 and the ureteric bud. Dev Genet. 1999;24:252-62.

3. Kreidberg JA, Sariola H, Loring JM, et al. WT-1 is required for early kidney development. Cell. 1993;74:679-91.

4. Carmona R, González-Iriarte M, Pérez-Pomares JM, Muñoz-Chápuli R. Localization of the Wilm's tumor protein WT1 in avian embryos. Cell Tissue Res. 2001;303:173-86.

5. Thiery JP, Sleeman JP. Complex networks orchestrate epithelial-mesenchymal transitions. Nat Rev Mol Cell Biol. 2006;7:131-42.

6. Aberle H, Schwartz H, Kemler R. Cadherin-catenin complex: protein interactions and their implications for cadherin function. J Cell Biochem. 1996;61:514-23.

7. Nieto MA. The snail superfamily of zinc-finger transcription factors. Nat Rev Mol Cell Biol. 2002;3:155-66.

8. Murray SA, Carver EA, Gridley T. Generation of a Snail1 (Snai1) conditional null allele. Genesis. 2006;44:7-11.

9. De Craene B, van Roy F, Berx G. Unraveling signalling cascades for the Snail family of transcription factors. Cell Signal. 2005;17:535-47.

10. Boutet A, De Frutos CA, Maxwell PH, Mayol MJ, Romero J, Nieto MA. Snail activation disrupts tissue homeostasis and induces fibrosis in the adult kidney. EMBO J. 2006;25:5603-13. 
11. Yang Z, Rayala S, Nguyen D, Vadlamudi RK, Chen S, Kumar R. Pak1 phosphorylation of snail, a master regulator of epithelial-to-mesenchyme transition, modulates snail's subcellular localization and functions. Cancer Res. 2005;65:3179-84.

12. Zhou BP, Deng J, Xia W, Xu J, Li YM, Gunduz M, Hung MC. Dual regulation of Snail by GSK-3betamediated phosphorylation in control of epithelialmesenchymal transition. Nat Cell Biol. 2004;6:931-40.

13. Barrallo-Gimeno A, Nieto MA. The Snail genes as inducers of cell movement and survival: implications in development and cancer. Development. 2005;132:3151-61.

14. Rosivatz E, Becker I, Specht K, et al. Differential expression of the epithelial-mesenchymal transition regulators snail, SIP1, and twist in gastric cancer. Am J Pathol. 2002;161:1881-91.

15. Rosivatz E, Becker KF, Kremmer E, et al. Expression and nuclear localization of Snail, an E-cadherin repressor, in adenocarcinomas of the upper gastrointestinal tract. Virchows Arch. 2006;448(3):277-87.

16. Dominguez D, Montserrat-Sentis B, Virgos-Soler A, et al. Phosphorylation regulates the subcellular location and activity of the snail transcriptional repressor. Mol Cell Biol. 2003;23:5078-89.

17. Francí C, Takkunen M, Dave N, et al. Expression of Snail protein in tumor-stroma interface. Oncogene. 2006;25:5134-44.

18. Katoh M, Katoh M. Cross-talk of WNT and FGF signaling pathways at GSK3beta to regulate betacatenin and SNAIL signaling cascades. Cancer Biol Ther. 2006;5:1059-64.

19. Prozialeck WC, Lamar PC, Appelt DM. Differential expression of E-cadherin, N-cadherin and betacatenin in proximal and distal segments of the rat nephron. BMC Physiol. 2004;4:10. 